\section{A rare cause of intestinal obstruction due to an exophytic gastrointestinal stromal tumor of the small bowel}

\author{
ATAKAN SEZER • MEHMET ALI YAGCI • AHMET RAHMI HATIPOGLU • \\ IRFAN COSKUN・IRFAN CICIN • UFUK USTA • OSMAN TEMIZOZ
}

\author{
ATAKAN SEZER $(\square) \bullet$ \\ MEHMET ALI YAGCI • \\ AHMET RAHMI HATIPOGLU • \\ IRFAN COSKUN \\ Trakya Universitesi Hastanesi \\ Genel Cerrahi Anabilim Dalı \\ 22030 Edirne- Türkiye \\ Phone: 02842357641 \\ Fax: + 902842361074 \\ E-mail: atakansezer@hotmail.com
}

IRFAN CICIN

Department of Medical Oncology Trakya University School of Medicine

Edirne, Turkey

UFUK USTA

Department of Pathology Trakya University School of Medicine

Edirne, Turkey

OSMAN TEMIZOZ

Department of Radiology

Trakya University School of Medicine

Edirne, Turkey

\begin{abstract}
Introduction: Gastrointestinal stromal tumors constitute a distinct group of rare gastrointestinal tract tumors that originate from the interstitial cells of Cajal. These jejunoileal lesions are a rare cause of obstruction but can be associated with substantial morbidity.

Case: A 59-year-old woman presented to the emergency department with abdominal pain and distention. Physical examination revealed tenderness and rebound in right lower quadrant. Computed tomography revealed a mass in lower right quadrant. A 9x9x4 cm exophytic ileal mass was observed at exploration. Preoperative diagnose was a small bowel tumor and then segmental resection and primary anastomosis were performed. Histopathological investigation revealed spindle cells that stained strongly for C-117, consistent with a diagnosis of a malign gastrointestinal stromal tumor.

Conclusion: We conclude that exophytic small bowel gastrointestinal stromal tumors are rare lesions, which should be kept in mind by physicians among the diagnosis of small bowel obstructions in order to reduce substantial morbidity and mortality.
\end{abstract}

Key words: intestinal obstruction, gastrointestinal stromal tumor, small bowel, surgery

\section{Introduction}

Small-bowel tumors account for 1 to $5 \%$ of gastro-intestinal (Gl) tumors. Adenocarcinoma, carcinoid, stromal tumors, lymphoma, endocrine tumors, and metastases are the pathologic types of small bowel neoplasms. (1) Gastro- intestinal stromal tumors (GISTs) are a rare form of small bowel tumor most often located in the jejunum or ileum. GISTs are difficult to diagnose and are often advanced at the time of definitive treatment. The main symptoms are bleeding and obstruction, which is caused by the growing tumor. (2) In this paper, we report an unusual case of an exophytic GIST which originated from the small bowel causing right lower quadrant pain and intestinal obstruction treated with surgical intervention.

\section{Case Report}

A 59-year-old woman presented to the emergency department with abdominal pain and distention. Her medical history was unremarkable. There was no history of abdominal surgery and no external hernia on examination. Tenderness and rebound was found in the right 


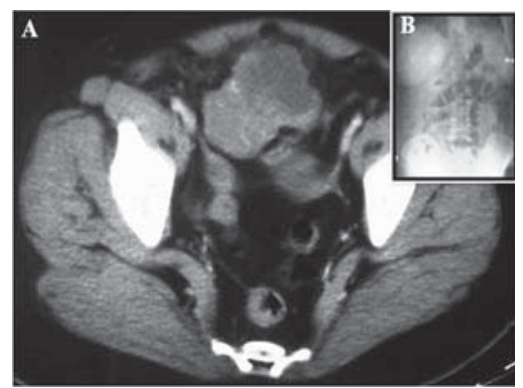

Figure 1. (A) Preoperative CT scans showing pelvic mass; Contrast enhanced CT scan showing lobulated $5 \times 7 \times 8 \mathrm{~cm}$ mass in the pelvis. (B) Plain abdominal $x$-ray showing air fluid levels.

lower quadrant which had been diagnosed as acute appendicitis previously. Initial laboratory examinations revealed normal values except for elevated white blood cell (WBC) count at 16,700/L. Plain abdominal $\mathrm{x}$-ray revealed dilated loops of small bowel. Computed tomography showed a lobulated mass $(5 \times 7 \times 8 \mathrm{~cm})$ in the lower right quadrant (figure 1). Computed tomography scan was suggestive of a soft tissue tumor arising from the small bowel. Based on the clinical and radiological findings, a diagnosis of small bowel obstruction secondary to a small bowel mass was made. The patient was subsequently referred for surgery. At laparotomy, an $8 \times 5 \times 7 \mathrm{~cm}$ firm, lobulated, pedunculated tumor was discovered arising from the anterior mesenteric border of the terminal ileum, approximately $50 \mathrm{~cm}$ from the ileocecal valve (figure 2). There was no evidence of distant spread. The tumor was excised with $5 \mathrm{~cm}$ of the ileum on both sides. End to end anastomosis was done in two layers. Macroscopic examination of the specimen revealed a predominantly solid tumor with hemorrhagic areas, which had no communication with the lumen. The histo-pathology report was that of a gastrointestinal stromal tumor arising from small bowel. The specimen contained spindle cells that stained strongly for CD-117, but desmin, actin, S100, and CD 34 were negative (figure 3). The patient made an uneventful recovery and remarkably, all her earlier abdominal symptoms com-

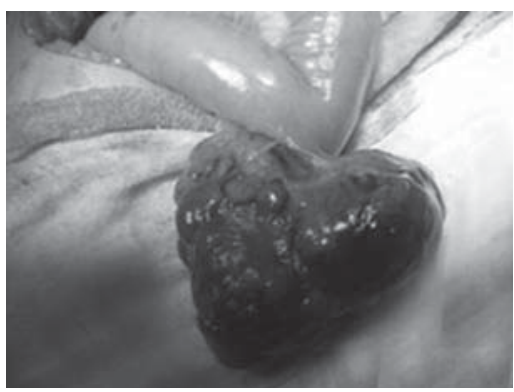

Figure 2. Intraoperative picture showing a pedunculated tumor arising from the ileum.

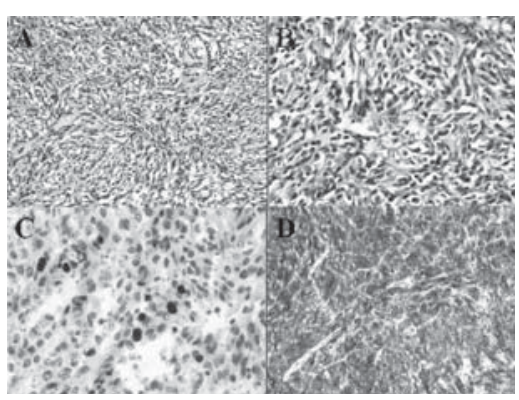

Figure 3. (A) Spindle and oval shaped tumor cells with clear and eosinophilic cytoplasm, arranged in fascicles and bundles (HEx100). (B) Higher view of the atypical cells with nuclei pleomorphism and prominent nucleoli (HEx200). (C) High proliferative index with Ki-67 staining the nuclei immunohistochemically (Ki-67×200). (D) Strong and diffuse cytoplasmic reaction for CD117 (C-kit) (CD117x100).

pletely resolved. The multidisciplinary oncology team decided not to proceed with any adjuvant treatment. At two years follow up the patient was completely free of disease.

\section{Discussion}

Tumors of the small intestine account for less than $1 \%$ of all gastrointestinal malignancies. GISTs arise anywhere within the gastrointestinal tract. Approximately, $70 \%$ of GISTs arise in the stomach, with $20-30 \%$ originating in the small intestine and the reminding $10 \%$ occurring in the esophagus, colon and rectum. $(1,2)$ GISTs predominantly occur in middle aged or older patients with a median age of sixty. Males and females are affected equally. They have distinctive histological and clinical fea- tures that vary according to their primary site of origin. The clinical presentation, which is variable and depends on the tumor size and anatomical site, consists of upper abdominal pain, fullness, gastrointestinal bleeding, and a palpable mass. (3) The vast majority of small bowel obstructions usually occur secondary to incarcerated hernias, invaginations, postoperative adhesions, or internal herniation but GISTs of the small intestine are the rarest cause of obstruction even in the presence of extensive peritoneal metastatic disease. $(4,5)$ On the other hand, GISTs arise from the muscularis mucosa or muscularis propria layers and most exhibit an endophytic growth pattern which may present with evidence of obstruction or bleeding. Exophytic tumors present as a large mass lesion or if perforation has occurred with evidence of peritonitis. (6) As in this case, the exophytic tumor may cause obstruction by kinking the small bowel due to traction by the large, primarily subserosal tumor. The diagnosis is generally based on symptoms. Plain radiography or barium studies may direct physicians to the proper diagnosis in the case of obstruction or large masses. On ultrasound GIST are seen as well defined mass lesions of different sizes. The echogenicity of the lesion depends on the size and intratumoral cystic or solid components of the tumor. The origin of the tumor is generally difficult to determine, unless there is ulceration with a pocket of gas in the ulcer crater, suggesting a tumor of the gut. Computed tomography or magnetic resonance investigation is useful in tumors larger than 2 centimeters. $(7,8)$ Endoscopic examinations fail to diagnose GISTs originated from the small intestine. GISTs are currently defined as CD-117 positive spindle cell or epithelioid neoplasms with minimal or incomplete myogenic or neural phenotype. $(4,9)$ As in our case, the specimen contained spindle cells that stained strongly for CD-117. These tumors are believed to be potentially malignant lesions with an average rate of 20-25\% of gastric and $40-50 \%$ of small intestinal localization. Metastases commonly 
develop in the abdominal cavity and liver; rarely, in bones, soft tissues, and skin. $(2,3,7,10)$ Surgical removal and resection are the only effective therapy and recommended in nearly all adult cases. The long term survival is $50 \%$ at best. Chemotherapy and radiotherapy do not increase the salvage rate. (2) In conclusion, the non-diagnostic physical findings and insidious presentation of the exophytic GISTs of the small intestine make a proper preoperative diagnosis generally elusive. These tumors may be the cause of mechanical small bowel obstruction due to external luminal compression and this rare entity should be kept in mind to reduce morbidity and mortality.

\section{REFERENCES}

1. Sunamak O, Karabicak I, Aydemir I, Aydogan F, Guler E, Cetinkaya S, et al. An intraluminal leiomyoma of the small intestine causing invagination and obstruction: a case report. Mt Sinai J Med 2006;73:1079-81.

2. Bucher $P$, Egger JF, Gervaz $P$, Ris F, Weintraub D, Villiger $P$, et al. An audit of surgical management of gastrointestinal stromal tumours (GIST). Eur J Surg Oncol 2006;32:310-4.

3. El Demellawy D, Shokry P, Ing A, Khalifa M. Polypoid gastrointestinal stromal tumor of small bowel metastasizing to mesenteric lymph nodes: a case report. Pathol Res Pract 2008;204:197-201.

4. Abbas M, Farouk Y, Nasr MM, Elsebae MM, Farag A, AkI MM, et al. Gastrointestinal stromal tumors (GISTs): clinical presentation, diagnosis, surgical treatment and its outcome. J Egypt Soc Parasitol 2008;38:883-94.

5. Beltran MA, Cruces KS. Primary tumors of jejunum and ileum as a cause of intestinal obstruction: a case control study. Int J Surg 2007;5:183-91.

6. Kimura H, Yoshida T, Kinoshita S, Takahashi I. Pedunculated giant gastrointestinal stromal tumor of the stomach showing extragastric growth: report of a case. Surg Today 2004;34:159-62.

7. Lupescu IG, Grasu M, Boros M, Gheorghe C, Ionescu M, Popescu I, et al. Gastrointestinal stromal tumors: retrospective analysis of the computer-tomographic aspects. J Gastrointestin Liver Dis 2007;16:147-51.

8. Spivach A, Zanconati F, Bonifacio Gori D, Pellegrino M, Sinconi A. Stromal tumors of the small intestine (GIST). Prognostic differences based on clinical, morphological and immunophenotypic features. Minerva Chir 1999;54:717-24.

9. Shah SN. Malignant gastrointestinal stromal tumor of intestine: a case report. Indian J Pathol Microbiol 2007;50:357-9.

10. Zanghi G, Di Stefano G, Strazzanti A, Benfatto G, Politi V, Basile F. Gastrointestinal stromal tumors: report of three cases and review of the literature. G Chir 2006;27:209-13. 